\title{
Apnea Recognition with Wavelet Neural Networks
}

\author{
C. ZANIOL $^{1}$, M.C. VARRIALE ${ }^{1}$ and E. MANICA ${ }^{2}$
}

Received on June 09, 2017 / Accepted on December 21, 2017

\begin{abstract}
Apnea is a Sleep Disorder Syndrome characterized by an interruption or reduction of air flow for at least 10 seconds. Polysomnography is a test used to apnea diagnosis. Several signals, including Electrocardiogram (ECG), Electroencephalogram (EEG) and Oxygen Saturation $\left(\mathrm{SpO}_{2}\right)$ are obtained in this diagnostic test. Since most tests for apnea are uncomfortable to the patients, there is an increase search for alternative methods to reduce cost and improve patient well-being. In this work, we use only $\mathrm{SpO}_{2}$ data from 25 patients of the St Vincent's University Hospital, Dublin, to extract parameters connected to a Neural Network to classify patients with apnea or non-apnea. Results confirm that our alternative method can be used as an auxiliary tool for diagnosis by using exclusively $\mathrm{SpO}_{2}$ signal.
\end{abstract}

Keywords: Neural Network, Sleep Disorder Syndrome, Apnea.

\section{INTRODUCTION}

On the past decades, the improvement in diagnosis of several diseases was ensured by the evolution of medical instrumentation and computational systems; also, the data volume to be processed has increased significantly $[6,11]$. Likewise, some studies have shown evidence of hidden information that cannot be evaluated visually or by traditional diagnostic methods [11].

In order to deal with increasing amount of information, and to optimize data analysis, computational methods are introduced to reduce the time spent by specialists, who would normally make the diagnosis just visually, as well to try granting better results. Computational methods have been shown useful in diagnosis of several diseases, such as epilepsy [9, 20], cardiac pathologies [1] and even image-based diagnosis, as mammography [5].

Sleep apnea is a disease with great impacts on individuals and to the health public system, characterized by an interruption or reduction of air flow for at least 10 seconds. Some comorbidities associated with sleep apnea include: depression, heart diseases, mellitus diabetes and obesity.

\footnotetext{
*Corresponding author: Cristina Zaniol - E-mail: criszaniol@gmail.com

${ }^{1}$ Programa de Pós Graduação em Matemática Aplicada, UFRGS, Av. Bento Gonçalves, 9500, 91509-900 Porto Alegre, RS, Brasil. E-mail: criszaniol@gmail.com; cris@mat.ufrgs.br

2 Instituto de Matemática e Estatística, UFRGS, Av. Bento Gonçalves, 9500, 91509-900 Porto Alegre, RS, Brasil. E-mail: evandro.manica@ufrgs.br
} 
In the United States, it is estimated that $13 \%$ of men and $6 \%$ of women suffer from some kind of sleep disorders [8]. In Brazil, there are few epidemiological studies. In [15], they showed that $11.5 \%$ of a sample of 209 drivers from São Paulo present evidence of apnea, whereas [23] detected moderate or severe apnea in $16.9 \%$ out of 1042 volunteers from São Paulo.

One important test for the diagnostic of sleep disorders is the Polysomnography. The sleep apnea using the Polysomnography, for instance, presents some difficulties, for instance: during this test, a patient stays the whole night in a clinic connected with electrodes which are uncomfortable and may add a lot of noise in the resulting data $[4,7]$. These data is analized visually by health care professionals in order to give the diagnosis to patients. This process, which underuses the given data, is subjective and adds a great deal of error to the diagnosis.

The first efforts on apnea pattern recognition were possible only after 2000, driven by the Challenge from PhysioNet and Computers in Cardiology 2000, whose objective was to verify the possibility of using ECG in apnea diagnosis $[11,21]$. This was the first step in finding alternative and better approaches to analyze data, using statistical and computational tools, as well as learning algorithms, such as Neural Networks. As well, wavelets have been applied on apnea diagnosis, which is a great tool for analyze non periodic and acyclic signals $[2,17,19]$.

It is well known that the Polysomnography is composed by several strongly correlated signals. In general, all signals are used to determine such patterns. Our purpose is to investigate if some of these signals may be helpful to classify whether a patient have or not apnea. In this work, we focus on only one of these signals: the Oxygen Saturation Signal $\left(\mathrm{SpO}_{2}\right)$ is used to evaluate apnea patterns of the data base UCDDB [22] (described in Section 4) and we use these results to compare to what is found in the literature $[4,7,8]$. This paper is organized as follows: Section 2, definition of Wavelets; Section 3, Neural Network description; Section 4, UCDDB data base and parameters acquisition; Section 5, some results; and, finally, Section 6, some final considerations and discussions.

\section{WAVELETS}

Wavelets are defined as a combination of orthogonal functions of the form [2, 19]

$$
\psi_{a, b}(t)=\frac{1}{\sqrt{a}} \psi\left(\frac{t-b}{a}\right), \quad a>0,
$$

where $a$ and $b$ are the scaling and the translation parameters, respectively. In the discrete form, a wavelet is written as

$$
\psi_{m, n}(t)=\frac{1}{a_{0}^{m / 2}} \psi\left(a_{0}^{-m} t-n b_{0}\right),
$$

where $m$ and $n$ have the roles of $a$ and $b$ in Equation (2.1), $a=a_{0}^{m}, m \in \mathbb{Z}$, and $a_{0} \neq 1$; and $b=n b_{0} a_{0}^{m}$, with $n \in \mathbb{Z}$. For simplicity, let $a_{0}>1$ and $b_{0}>0$. The wavelet coefficient (or detail coefficient) is given by

$$
T_{m, n}=\int_{-\infty}^{\infty} f(t) \psi_{m, n}(t) d t
$$


and the approximation coefficient by

$$
S_{m, n}=\int_{-\infty}^{\infty} f(t) \phi_{m, n}(t) d t
$$

The signal approximation in the scale $m$ is defined by

$$
f_{m}(t)=\sum_{n=-\infty}^{\infty} S_{m, n} \phi_{m, n}(t)
$$

and the detail,

$$
d_{m}(t)=\sum_{n=-\infty}^{\infty} T_{m, n} \psi_{m, n}(t)
$$

As a consequence, the signal can be rewritten in a multiresolution representation, as a sum of the signal approximation in a given scale and details

$$
f(t)=f_{m_{0}}(t)+\sum_{m=-\infty}^{m_{0}} d_{m}(t)
$$

Considering that $d_{m}$ is related to the high frequency components and $f_{m}$ to the low frequency components, the transformation of the signal using the parameters $m$ e $n$ allows the $f(t)$ decomposition in both high and low frequency components $[2,17]$. Thus, wavelet enables one to study different band frequencies separately, as necessary for describing characteristics of the signal $[3,19]$.

In this work, we will use the Daubechies family of wavelets. Daubechies wavelets are a family of orthogonal wavelets with compact support and do not have an explicit form [14]. These wavelets have been used in numerous biomedical signals, including ECG, EEG, and in pattern recognition of apnea.

\section{NEURAL NETWORK}

Neural Networks are learning algorithms capable of generalizing patterns used in classification [12]. Based on $\mathrm{N}$ interconnected neurons, the $k^{\text {th }}$ neuron in a Neural Network, $k=1,2, \ldots, N$, can be represented as

$$
u_{k}=\sum_{j=1}^{m} w_{k j} x_{j}
$$

where $w_{k j}$ is the weight of a given $j^{\text {th }}$ input signal $x_{j}, j=1,2, \ldots, m$. The output $y_{k}$ of Neural Network generated by the $k^{\text {th }}$ neuron is given by

$$
y_{k}=\sigma\left(v_{k}\right)
$$

where

$$
v_{k}=u_{k}+b_{k}
$$


with $b_{k}$ being a bias factor which allows adjust the data according to the expected result, or in other words, it could be improved efficiency in training; and $\sigma(\cdot)$, the activation function, based on the functioning of synapses, inhibits or activates transmission of information when a given threshold is exceeded. In this work, we use the logistic sigmoid function, defined as

$$
\sigma\left(v_{k}\right)=\frac{1}{1+e^{\lambda v_{k}}}
$$

where $\lambda$ is a slope factor.

There are two important characteristics of a Neural Network that we must consider: the learning paradigm and the topology. The learning paradigm is related to the way we use the data to describe the patterns in dataset: if dataset was previously classified by specialists, one could use this information in the Neural Network learning (Supervised Learning); otherwise, one could properly group data for a better description (Unsupervised Learning). Topology is related with the neuronal structure: the number of neurons and how they are connected.

There is no theory to determine how many hidden neurons are necessary to approximate output to a known classification $[12,13]$. The process to choose how many neurons is empirical, but we know that few hidden neurons increase training error and cannot generate a good generalization, that is, we have an under-fitting; on the other hand, if we have an excessive number of hidden neurons, we incur in a low training error, but the overfitting does not allow to generalize information.

In this work, we use supervised learning. We choose a topology $\mathrm{P}-\mathrm{N}-2$, where $\mathrm{P}$ is the number of input parameters and $\mathrm{N}$, the number of neurons in the hidden layer of Neural Network, which we vary from 5 to 50 neurons. The learning algorithm employed was the SCG (Scaled Conjugate Gradient), with MSE-reg (Mean Square Error Regularized) [18], present in Matlab R2012b. To evaluate the training result, we use leave-one-out cross-validation: $T-1$ patients are used for training and the $T^{\text {th }}$ patient is used to evaluate the result of the training.

Sensitivity, Specificity and Accuracy are used to measure the precision of pattern recognition. Each one of them represents a proportion of, respectively: correct apnea classification, correct non-apnea classification and total correct classification. These measures are given by [7]

$$
\begin{array}{r}
\text { Sensitivity }=\frac{T P}{T P+F N}, \\
\text { Specificity }=\frac{T N}{T N+F P}, \\
\text { Accuracy }=\frac{T P+T N}{T P+F P+T N+F P},
\end{array}
$$

where TP (True Positive) and TN (True Negative) describe the correct pattern recognition; FP (False Positive) and FN (False Negative), the incorrect pattern recognition. Obviously, our goal is to achieve the highest value for the three parameters described above. 


\section{THE DATA AND THE PARAMETER EXTRACTION}

A dataset with information from 25 patients of St Vincent's University Hospital, Dublin, with suspicion of Sleep Disorder Syndrome was used for training and testing our Neural Network $[11,22]$. The patients were between 28 and 68 years old, wherein 4 women and 21 men, and, in average, overweight (normal Body Mass Index - BMI - is between 18.5 and 24.9), as can be observed in the Table 1. Also, no patient among the subjects had known heart disease or autonomic dysfunction, responsible for the blood flow decrease in muscular and connective tissues, and they did not use medicines that interfere in heart frequency.

Table 1: Age, BMI and AHI of analyzed Polysomnography.

\begin{tabular}{ccccccccc}
\hline & Age & \multicolumn{4}{c}{ BMI } & \multicolumn{3}{c}{ AHI } \\
\hline Mean & Min & Max & Mean & Min & Max & Mean & Min & Max \\
\hline 50 & 28 & 68 & 31.6 & 25.1 & 42.5 & 24.24 & 2 & 91 \\
\hline
\end{tabular}

As shown in Table 1, patients are, in average, 50 years old and obese. The Apnea-Hypopnea Index (AHI), presented in the third column, describes the apnea severity: a healthy patient presents between 0 and 4 apneas hourly; in the range of 5 to 14, mild sleep apnea; between 15 and 29, moderate sleep apnea; and above 30, severe sleep apnea. Even having average AHI characterizing moderate sleep apnea, some patients present normal sleep apnea and others, severe sleep apnea. This is important in order to the Neural Network learn the various patterns present in the dataset.

The exams were performed at night, lasting between 5 and 8 hours, using Jaeger-Roennies system. Electroencephalography (EEG), Electrooculography (EOG), Electromyography (EMG), Electrocardiography (ECG), nasal airflow, abdominal and thoracic effort, oxygen saturation $\left(\mathrm{SpO}_{2}\right)$, noise and corporal position are the signals that compose Polysomnography.

For the purpose of this work, we use only $\mathrm{SpO}_{2}$. Data are collected by an external device (Oximeter), which is connected to a finger and estimates oxygen saturation in the patient blood. Normal values for oxygen saturation in the blood are superior to $90 \%$, but, in apnea events, it could be less than $70 \%$ [10]. Errors in data extraction come from several sources and, among them, we can cite machine inaccuracies and human errors. Thus, to avoid this problem, the data must undergo to a preprocessing stage, where we use a median filter to attenuate these errors [16].

For signal analysis and parameter extraction, we subdivide the signal in segments. Each one of them lasting 60 seconds. For this analysis, we estimate the following parameters: mean, minimum value, number of $\mathrm{SpO}_{2}<92 \%$, mean of variation and variation from the mean, based on [4], that we will call $\mathrm{SpO}_{2}$ parameters. Additionally, we performed the wavelet decomposition in 8 scales using Daubechies Wavelet of order 4, which generate other parameters to feed the Neural Network: for each decomposition level, we calculate mean, standard deviation, entropy, skewness and kurtosis, as will be described in the follow section. 


\section{RESULTS}

We perform four tests in this work: Test A, based on $\mathrm{SpO}_{2}$ parameters; Test $\mathrm{B}$, that uses wavelets parameters explained below; Test $\mathrm{C}$, which combines both $\mathrm{SpO}_{2}$ and wavelets parameters; and Test D, based on [7]. We start by comparing our results (Specificity, Sensibility and Accuracy) among themselves, then we compare with other results in literature. The results are displayed on a box plot: a graph that shows the median (the red line inside of the box) and the variability outside the upper and lower quartiles (dashed line outside box) from the distribution of the results. For each test, the accuracy of $N=5,10,15,20,25,30,35,40,45$ and 50 neurons on the hidden layer is presented on Figures 1, 2, 3 and 4, respectively; and the corresponding average results from sensitivity, specificity and accuracy are tabulated on Tables 2, 3, 4 and 6. Note that each point in the box plot represents the accuracy to each test after training, using leave-one-out crossvalidation: for all $N$ neurons, we use 24 patient for training and the last one is left to verify the accuracy: thus, we considered 25 tests in total for each $\mathrm{N}$ neurons on the hidden layer.

For Test A, the box plots in Figure 1 represent the accuracy for each number of neurons on the hidden layer of our Neural Network; Table 2 displays the respective sensitivity, specificity and accuracy results.

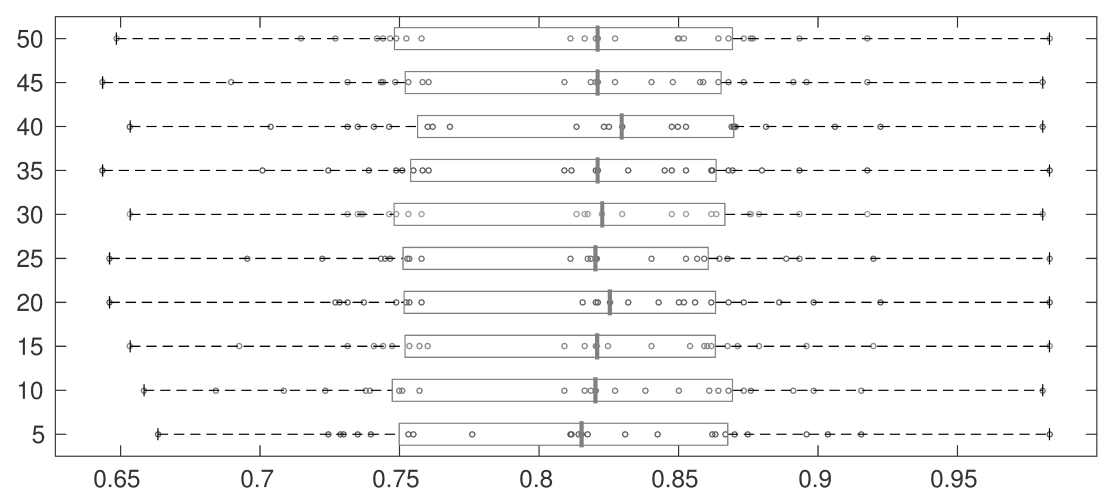

Figure 1: Test A: Accuracy of $\mathrm{N}$ neurons layer, considering only $\mathrm{SpO}_{2}$ parameters.

Table 2: Test A: average results in \%.

\begin{tabular}{ccccccccccc}
\hline Neurons & 05 & 10 & 15 & 20 & 25 & 30 & 35 & 40 & 45 & 50 \\
\hline Sensitivity & 33.12 & 35.32 & 38.63 & 37.48 & 37.92 & 36.16 & 37.48 & 36.75 & 37.99 & 38.06 \\
Specificity & 94.06 & 93.90 & 92.80 & 92.56 & 92.76 & 92.67 & 92.75 & 92.66 & 93.26 & 91.99 \\
Accuracy & 81.55 & 82.20 & 82.19 & 82.62 & 81.99 & 82.33 & 82.44 & 82.82 & 82.28 & 82.28 \\
\hline
\end{tabular}


From Figure 1, we observe that the best median accuracy was approximately 83\%, corresponding to 40 neurons, and most of the accuracy values are between 80 and $85 \%$. Also, we are able to visualize the absence of outliers, and the minimum accuracy was roughly in $65 \%$. In Table 2 , we can see, for each $\mathrm{N}$, average specificity, sensitivity and accuracy; the latter refers to the data analyzed in Figure 1. Observe that the average specificity is greater than $90 \%$ for all cases; besides, the average sensitivity is greater than $30 \%$, with the best average sensitivity when using 15 neurons, $38.63 \%$. This way, our tests indicate that if we are to aim for better sensitivity results, we could choose 15 neurons; but the higher accuracy is achieved with 40 neurons, $82.82 \%$.

In Test B, we decompose the normalized fragments of the signal in 8 scales using Daubechies Wavelet of order 4. For each decomposition and for the approximation, we consider as parameters: mean, standard deviation, entropy, skewness and kurtosis, totalizing 45 parameters to be employed in the Neural Network. The box plot of the obtained accuracy is presented in Figure 2, where we exclude one outlier for 30 neurons, with accuracy under 20\%; in Table 3, the average specificity, sensitivity and accuracy for each $\mathrm{N}$ neurons.

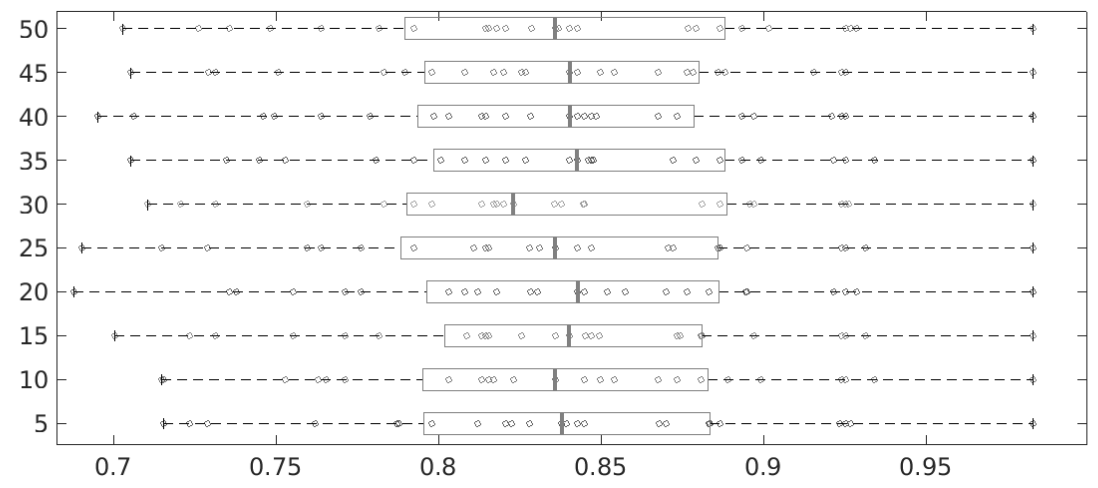

Figure 2: Test B: Accuracy of $\mathrm{N}$ neurons layer, considering wavelet parameters.

Table 3: Test B: average results in $\%$.

\begin{tabular}{ccccccccccc}
\hline Neurons & 5 & 10 & 15 & 20 & 25 & 30 & 35 & 40 & 45 & 50 \\
\hline Sensitivity & 43.82 & 41.97 & 42.73 & 42.68 & 42.85 & 40.24 & 42.65 & 39.52 & 42.41 & 41.45 \\
Specificity & 90.98 & 91.68 & 91.29 & 91.45 & 91.03 & 92.87 & 91.89 & 91.65 & 91.40 & 91.87 \\
Accuracy & 83.69 & 83.73 & 83.72 & 83.75 & 83.47 & 83.62 & 84.00 & 83.31 & 83.66 & 83.62 \\
\hline
\end{tabular}

Note that, in Figure 2, the median accuracies are closer to $85 \%$ than Test A. Further, we could see the accuracy of the results are concentrated around the median, which indicates a more stable accuracy. Also, in Table 3, we have an improvement in average sensitivity, from about 30\% to $40 \%$, displaying a better recognition of true apnea events; moreover, the average specificity is the 
same or at most $4 \%$ smaller than from Test A. As consequence, we get a better average accuracy results.

Lastly, in Test C, we combine $\mathrm{SpO} \mathrm{O}_{2}$ parameters and $\mathrm{SpO}$ 2 wavelet parameters. We were working to improve results adding more parameters, and therefore information, for the Neural Network. The results are presented in Figure 3 and in Table 4.

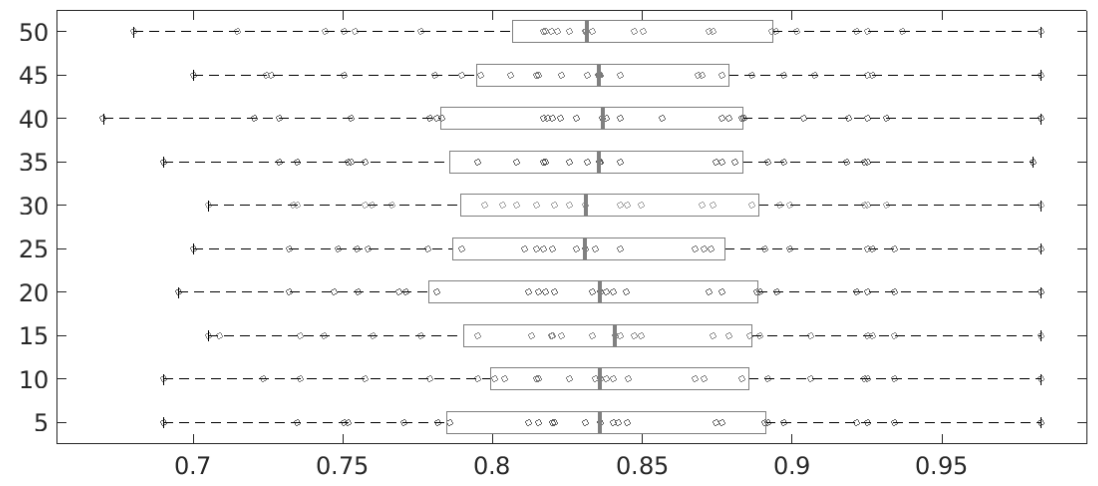

Figure 3: Test C: Accuracy of $\mathrm{N}$ neurons layer, considering $\mathrm{SpO}_{2}$ and wavelet parameters.

Table 4: Test C: average results in \%.

\begin{tabular}{ccccccccccc}
\hline Neurons & 5 & 10 & 15 & 20 & 25 & 30 & 35 & 40 & 45 & 50 \\
\hline Sensitivity & 41.84 & 43.08 & 40.77 & 41.66 & 42.24 & 39.24 & 41.91 & 42.60 & 43.10 & 42.80 \\
Specificity & 91.85 & 90.97 & 92.19 & 91.90 & 91.56 & 91.92 & 91.09 & 91.45 & 91.06 & 91.60 \\
Accuracy & 83.69 & 83.68 & 83.67 & 83.57 & 83.49 & 83.54 & 83.35 & 83.52 & 83.40 & 83.66 \\
\hline
\end{tabular}

In Test $\mathrm{C}$, the results were similar to Test $\mathrm{B}$ and better than results from Test $\mathrm{A}$. The use of all parameters is not able to improve in most of the neurons numbers. As a remark, observe that the variance on accuracy results for any number of neurons used in this work has increased, as can be seen in Figure 3.

For better comparison, we performed a fourth test, Test D, based on [7], originally performed on a different dataset (from children of Clinical Hospital of Catholic University of Chile), using Wavelet Transform and Neural Network for pattern recognition. The difference lies in the set of parameters (mean, variance and energy) and the wavelet decomposition: while we divided on 8 scales, [7] divided on 14 scales; we both used Daubechies 4 and Neural Network. It is important to note that we performed the classification with our Neural Network on our dataset [22] because we do not have neither information about the Neural Network used by [7], nor access to the dataset of Clinical Hospital of Catholic University of Chile. Results are presented on Figure 4 and Table 5. 


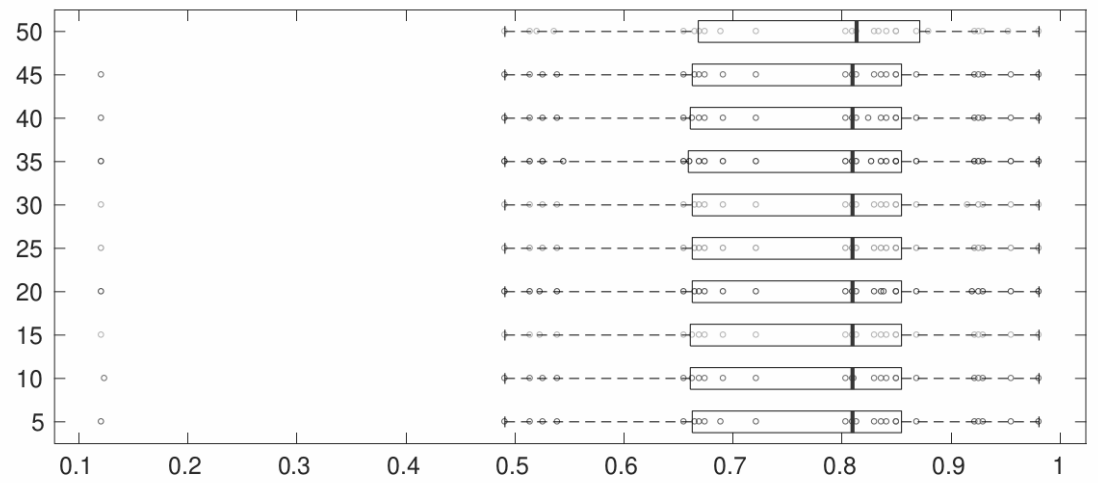

Figure 4: Test D: Accuracy of N neurons layer, based on parameters of [7].

Table 5: Test D: average results in \%.

\begin{tabular}{ccccccccccc}
\hline Neurons & 5 & 10 & 15 & 20 & 25 & 30 & 35 & 40 & 45 & 50 \\
\hline Sensitivity & 0.00 & 0.01 & 0.00 & 0.00 & 0.00 & 0.00 & 0.13 & 0.00 & 0.00 & 4.00 \\
Specificity & 99.99 & 99.97 & 99.96 & 99.96 & 100.00 & 99.97 & 99.89 & 99.96 & 100.00 & 95.90 \\
Accuracy & 73.90 & 73.91 & 73.89 & 73.88 & 73.91 & 73.89 & 73.91 & 73.88 & 73.91 & 76.89 \\
\hline
\end{tabular}

Although the median accuracies of Figure 4 are near $80 \%$, the average accuracies is about $70 \%$; this fact can be explained by the existence of outliers, with accuracy near $10 \%$. Also, we can note that Test $\mathrm{D}$ is not able to recognize apnea events, just non apnea.

In Table 6, we present the columns corresponding to the best and worst accuracies in Tables 2, 3, 4 and 5. Observe that Test $\mathrm{B}$ and Test $\mathrm{C}$ have higher sensitivity than Test A; no significant differences are detected with regard to their specificity. Accuracy has some improvement in Test $\mathrm{B}$ and Test $\mathrm{C}$, because sensitivity in Test $\mathrm{A}$ is smaller than the others and specificity has smaller influence on accuracies results in this case.

Table 6: Average results of pattern recognition for each test (in \%).

\begin{tabular}{ccccccccc}
\hline & \multicolumn{2}{c}{ Test A } & \multicolumn{2}{c}{ Test B } & \multicolumn{2}{c}{ Test C } & \multicolumn{2}{c}{ Test D } \\
\cline { 2 - 9 } & Min Acc & Max Acc & Min Acc & Max Acc & Min Acc & Max Acc & Min Acc & Max Acc \\
\hline Neurons & 5 & 40 & 40 & 35 & 35 & 5 & 20 and 40 & 50 \\
Sensitivity & 33.12 & 36.75 & 39.52 & 42.65 & 41.91 & 41.84 & 0.00 & 4.00 \\
Specificity & 94.06 & 92.66 & 91.65 & 91.89 & 91.09 & 91.85 & 99.96 & 95.90 \\
Accuracy & 81.55 & 82.82 & 83.31 & 84.00 & 83.35 & 83.69 & 73.88 & 76.89 \\
\hline
\end{tabular}

Min Acc: Minimum Accuracy; Max Acc: Maximum Acurracy. 
We compare our results with [4] and [7], as showed in Table 7. For the comparison, we consider our worst and best average accuracy results on all tests, that is, 5 neurons on Test A and 35 neurons on Test B, respectively. For comparison with [7], we use the best result obtained on Test D.

In [4], apnea recognition was performed with the same data base, UCDDB [22], and the same parameter set. The difference was on the classification procedure: while our classification derives from Neural Network, they used Logistic Discriminant Function as a classification tool [4].

Table 7: Comparison between our results with [4] and [7] (in \%).

\begin{tabular}{ccccc}
\hline & Min Acc $^{1}$ & Max Acc $^{2}$ & Test D $^{3}$ & de Chazal et al., 2015 \\
\hline Sensitivity & 33.12 & 42.65 & 4.00 & 37.50 \\
Specificity & 94.06 & 91.89 & 95.90 & 79.00 \\
Accuracy & 81.55 & 84.00 & 73.88 & 70.60 \\
\hline
\end{tabular}

Min Acc: Minimum Accuracy; Max Acc: Maximum Acurracy.

${ }^{1}$ Worst accuracy of Test A; ${ }^{2}$ Best accuracy of Test B; ${ }^{3}$ Best result of Test D.

In comparison with [4], sensitivity was smaller for $\mathrm{SpO}_{2}$ parameters and slightly better when we added wavelet parameters. We note, however, that in Tests A, B and C, we were able to obtain higher specificity, achieving better accuracy. In this way, we observed that Neural Networks delivered better results than Logistic Discriminant Function. It is needed further studies to determine what makes our results better with Neural Network with the same parameter set, but we speculate that one of the reasons may be the linear combination of parameters on Logistic Discriminant Function, in contrast with Neural Network that learn from a fuzzy and complex combination of parameters.

Concerning [7], since we are considering fewer parameters than Test A, B and C, Neural Network have a smaller set of characteristics to classify non-apnea, thus specificity and accuracy were lower than our previous results. Anyhow, we obtain better results with our parameters, both related to specificity and sensitivity, in which accuracy differs in, at least, $10 \%$.

We remark that a bigger dataset could generate better results, because more patterns imply a bigger learning capability.

\section{CONCLUDING REMARKS}

Our results in this study indicate that Oxygen Saturation $\left(\mathrm{SpO}_{2}\right)$ evaluation could aid in diagnosis, since it supports patients exclusion with mild or light apnea. More studies are required to determine parameters and better Wavelet Transform that could increase diagnosis effectiveness, as well as other Neural Network topologies. Another difficulty is the selection and the definition of the ideal number of parameters to not incur in overtraining. 
Even though we are able to obtain accuracy higher than $80 \%$, specificity only ensures the use of our method as an auxiliary tool to traditional diagnosis. Thus, we suggest our method as exclusion tool of normal patients, being necessary other analyzes in Polysomnography to discover severity or confirm a diagnosis.

As future study, we could list the application of our algorithm on a bigger data base, with patients of south of Brazil, that could allow the access on a huge number of patterns and, possibly, a better accuracy in apnea pattern recognition.

\section{ACKNOWLEDGMENT}

\section{Thanks to CAPES.}

RESUMO. Apneia é uma Desordem do Sono caracterizada pela interrupção ou redução do fluxo de ar por pelo menos 10 segundos. Seu diagnóstico é realizado principalmente com a Polissonografia, exame composto por uma série de sinais como o Eletrocardiograma (ECG), Electroencefalograma (EEG) e Saturação do Oxigênio $\left(\mathrm{SpO}_{2}\right)$. A maioria dos exames para o diagnóstico da apneia são desconfortáveis e caros, incentivando a busca por métodos alternativos tanto para a redução do custo, quanto para o aumento do bem-estar do paciente. Neste trabalho, foi utilizado apenas dados advindos do $\mathrm{SpO}_{2}$ de 25 pacientes do St Vincent's University Hospital, Dublin, para extrair parâmetros e alimentar uma Rede Neural, que realiza a classificação de pacientes com apneia. Os resultados confirmam que o método apresentado pode ser usado como uma ferramenta auxiliar no diagnóstico.

Palavras-chave: Redes Neurais, Doenças do Sono, Apneia.

\section{REFERENCES}

[1] M.M. Baig \& H. Gholamhosseini. Smart Health Monitoring Systems: an Overview of Design and Modeling. Journal of medical systems, 2(37) (2013), 1-14.

[2] C.K. Chui \& Q. Jiang. "Applied Mathematics: Data Compression, Spectral Fourier Analysis, Wavelets, and Applications", Mathematics Textbooks fo and Engineering. Atlantis Press (2013).

[3] I. Daubechies. "Ten lectures on wavelets", volume 61. SIAM (1992).

[4] P. de Chazal, N. Sadr \& M. Jayawardhana. An ECG Oximetry System for Identifying Obstructive and Central Apnoea Events. In "37th Annual International Conference of the IEEE". Engineering in Medicine and Biology Society (EMBC) (2015), pp. 7671-7674.

[5] J. Dheeba, N.A. Singh \& S.T. Selvi. Computer-aided Detection of Breast Cancer on Mammograms: A Swarm Intelligence Optimized Wavelet Neural Network Approach. Journal of Biomedical Informatics, (49) (2015), 45-52.

[6] I. Dimitrovski, D. Kocev, I. Kitanovski, S. Loskovska \& S. Deroski. Improved Medical Image Modality Classification Using a Combination of Visual and Textual Features. Computerized Medical Imaging and Graphics, 39 (2015), 14-26. 
[7] L. Erazo \& S.A. Riós. A Benchmark on Automatic Obstructive Sleep Apnea Screening Algorithms in Children. Procedia Computer Science, 35 (2014), 739-746.

[8] H. Espiritu \& V. Metsis. Automated Detection of Sleep Disorder-related Events from Polysomnographic Data. In "International Conference on IEEE". Healthcare Informatics (ICHI) (2015), pp. 562-569.

[9] O. Faust, U.R. Acharya, H. Adeli \& A. Adeli. Wavelet-based EEG Processing for Computer-aided Seizure Detection and Epilepsy Diagnosis. Seizure, (26) (2015), 56-64.

[10] W.W. Flemons \& W.T. McNicholas. Clinical Prediction of the Sleep Apnea Syndrome. Sleep Medicine Reviews, 1(1) (1997), 19-32.

[11] A.L. Goldberger, L.A. Amaral, L. Glass, J.M. Hausdorff, P.C. Ivanov, R.G. Mark, J.E. Mietus, G.B. Moody, C.K. Peng \& H.E. Stanley. Physiobank, Physiotoolkit, and Physionet Components of a New Research Resource for Complex Physiologic Signals. Circulation, 101(23) (2000), 215-220.

[12] S. Haykin. "Neural Networks and Learning Machines", volume 10. Prentice Hall (2009).

[13] G.B. Huang, L. Chen \& C.K. Siew. Universal Approximation Using Incremental Constructive Feedforward Networks with Random Hidden Nodes. IEEE Transactions on Neural Networks, 17(4) (2006), 879-892.

[14] G. Kaiser. "A friendly guide to wavelets", Springer Science and Business Media (2010).

[15] L.C. Lemos, E.C. Marqueze, F. Sachi, G. Lorenzi-Filho \& C.R.C. Moreno. Síndrome da Apneia Obstrutiva do Sono em Motoristas de Caminhão. Jornal Brasileiro Pneumologia, 35(6) (2009), 500-506.

[16] J.S. Lim. "Two-dimensional Signal and Image Processing”, volume 1. Englewood Cliffs, NJ, Prentice Hall (1990), pp. 469-476.

[17] S. Mallat. "A wavelet tour of signal processing”. The Spa Academic Press, 3rd edition (2008).

[18] M.F. Møller. A Scaled Conjugate Gradient Algorithm for Fast Supervised Learning. Neural Networks, 6(4) (1993), 525-533.

[19] Y. Nievergelt. "Wavelets Made Easy". Birkhauser (1999).

[20] V.P. Nigam \& D. Graupe. "A Neural-network-based Detection of Epilepsy". Neurological Research (2013).

[21] T. Penzel, J. McNames, P.D. Chazal, B. Raymond, A. Murray \& G. Moody. Systematic Comparison of Different Algorithms for Apnoea Detection Based on Electrocardiogram Recordings. Medical and Biological Engineering and Computing, 40(4) (2002), 402-407.

[22] U. St. "Vincent's University Hospital/University College Dublin Sleep Apnea Database" (2008). URL http://physionet.org/physiobank/database/ucddb/.

[23] S. Tufik, R. Santos-Silva, J.A. Taddei \& L.R.A. Bittencourt. Obstructive Sleep Apnea Syndrome in the Sao Paulo Epidemiologic Sleep Study. Sleep Medicine, 11(5) (2010), 1389-9457. 